\title{
„Wichtig sind für uns vor allem die Leitlinien, bei denen es große Unsicherheit bezüglich der Auswahl der geeigneten Bildgebung gibt.“
}

Im regen Austausch mit den radiologischen Kolleginnen und Kollegen sowie den Zuweiserinnen und Zuweisern aus den pädiatrischen Fächern erarbeitet die Gesellschaft für Pädiatrische Radiologie eine Vielzahl an Leitlinien. Welche davon besonders wichtig sind und wie die GPR ihre Leitlinienarbeit organisiert, erörtert Prof. Dr. Hans-Joachim Mentzel, Präsident der GPR, im Interview.

\section{An wie vielen Leitlinien ist die GPR aktuell} beteiligt?

Die GPR ist derzeit an 91 Leitlinien beteiligt, die bei der AWMF angemeldet sind.
Gibt es hier Projekte, die Sie eigens erwähnen möchten?

Wichtig sind für uns vor allem die Leitlinien, bei denen es große Unsicherheit bezüglich der Auswahl der geeigneten Bildgebung gibt. Aus diesem Grund existieren weiterhin unsere zwölf S1-Leitlinien, die sich symptombezogen vorrangig an die radiologischen Abteilungen und Institute richten, in denen keine Kinder- und Jugendradiologinnen und -radiologen arbeiten. Neben den Aspekten des Strahlenschutzes ist es vorrangig die Vermeidung einer teilweise noch üblichen Stufendiagnostik; es soll das Verfahren mit der besten Aussagekraft und

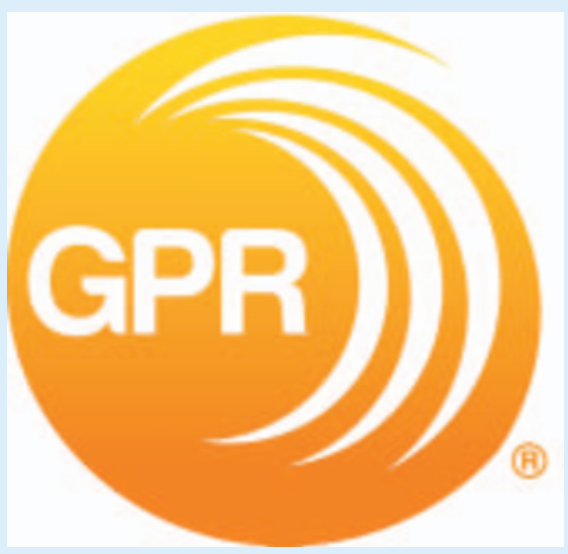

den geringsten negativen Effekten für das Kind ausgewählt werden. 
Wichtig sind für uns zudem die großen interdisziplinären Leitlinien, die Kernprobleme der Kinder- und Jugendmedizin betreffen und bei denen die Bildgebung eine große Rolle spielt - zum Beispiel das Polytrauma oder das isolierte Schädel-HirnTrauma im Kindes- und Jugendalter. Hier gab es in den letzten Jahren eine deutliche Dynamik bezüglich der Auswahl der Methoden in der Primär- und Folgediagnostik.

Wie organisieren Sie die Leitlinienarbeit intern innerhalb der GPR, aber auch in der externen Kooperation mit der DRG oder anderen pädiatrischen Disziplinen?

In der GPR gibt es eine Leitlinienkommission, die aus 10 Kolleginnen und Kollegen besteht. Wichtig ist für uns, dass sich dieses Gremium aus Vertreterinnen und Vertretern der ambulanten und stationären Kinder- und Jugendradiologie zusammensetzt, die Mitglieder also sowohl aus radiologischen Praxen, Kinderkrankenhäusern und Universitätskliniken kommen. Die Kommission trifft sich regelmäßig zum Austausch über die Leitlinien - in den letzten Jahren in zunehmender Frequenz auf digitalem Weg. Mit der DRG, der DeGIR und der DGNR gibt es ein gemeinsames Leitlinien- register - hier existiert reger Austausch über die Leitlinieninhalte und die jeweiligen Mandatsträger. Mit den Fachgesellschaften der konservativen und operativen Kinderund Jugendmedizin und deren Leitlinienkommissionen besteht über den Konvent der pädiatrischen Fachgesellschaften reger Austausch.

\section{Initiieren Sie auch selbst Leitlinien?}

Die GPR hat einige der oben erwähnten S1-Leitlinien initiiert, die sie gemeinsam mit der DRG bearbeitet und die schon seit einigen Jahren immer wieder aktualisiert werden. Diese „Auffrischungen“ sind erforderlich, da insbesondere in der Bildgebung häufig modernere Verfahren Anwendung finden, die auch in den Leitlinien Berücksichtigung finden sollen. Beispielhaft sei die Anwendung von Ultraschallkontrastmitteln genannt. Neuere eigene Leitlinien der GPR beschäftigen sich mit der GanzkörperMRT bzw. mit der gezielten Trauma-Diagnostik.

Welche Bedeutung hat die Leitlinienarbeit - für das Fach Kinderradiologie aber auch für die tägliche kinderradiologische Praxis?
Für die Kinder- und Jugendradiologie ist die Leitlinienarbeit sehr wichtig. Die Beteiligung unserer Fachgesellschaft an Leitlinien spiegelt letztlich die Relevanz unseres Faches wider und ist Ausdruck der Anerkennung der Arbeit der Kinder- und Jugendradiologinnen und -radiologen. Für die tägliche Praxis sind die Leitlinien immer etwas, worauf wir uns in der Durchführung unserer Untersuchung berufen können. Insbesondere dann, wenn wir als Radiologinnen und Radiologen zum Beispiel bei der Auswahl oder Durchführung von Untersuchungen andere Vorstellung als die Zuweiserinnen und Zuweiser haben, sind Leitlinien ein gutes Instrument zur Rechtfertigung unseres Vorgehens. Wichtig ist aber immer, dass Leitlinien keinen Richtliniencharakter haben und wir natürlich individuell entscheiden, wie im konkreten Einzelfall vorzugehen ist - gegebenenfalls auch abweichend von den Vorgaben der Leitlinie. Was allerdings stets gut begründet sein muss.

Leitlinien mit GPR-Beteiligung auf einen Blick:

www.kinder-radiologie.org > Leitlinien 